\title{
Seasonal fluctuation of freshwater ascomycetes in Nile Delta region (Egypt)
}

Ahmed M. Abdel-Raheem ${ }^{1}$, Gamalat A. Abd-Elaah ${ }^{1}$, Osman M. El-Maghraby ${ }^{1}$ and Mahmoud S. Bakhit $^{1}$

${ }^{1}$ Botany department, Faculty of Science, Sohag University.

Rec. 5 Apr, 2014 Accept. 3 May, 2014

\begin{abstract}
Seasonality of freshwater ascomycetes was studied in Nile Delta region from February 2010 to December 2011. One hundred and ten ascomycetes were identified through this investigation. The most common ascomycetes recovered during fluctuation study of Delta region were Zopfiella latipes, Microthelia sp. 1, Helicascus aegyptiacus and Naïs aquatica. Physico-chemical analysis (Water temperature, $\mathrm{pH}$, concentration of $\mathrm{NH}_{3}, \mathrm{NO}_{2}^{-}$and $\mathrm{PO}_{4}{ }^{3-}$ ) were recorded and had significant effect during this study.
\end{abstract}

Key word: Freshwater, ascomycetes, Delta region.

\section{Introduction}

Aquatic fungi are universally distributed occurring from Arctic to tropical waters (Jones and Pang, 2012). Freshwater fungi comprise a cosmopolitan and phylogenetic diverse group (McLaughlin et al., 2001) and consist mostly of microfungi, including meiosporic ascomycetes and mitosporic ascomycetes (Shearer, 1993, 2001). Freshwater ascomycetes are an ecological assemblage of fungi that occur on submerged or partially submerged substrates in aquatic habitats (Shearer, 2001; Vijaykrishna et al., 2006). Some freshwater ascomycetes are cosmopolitan in distribution, while others are reported only from their type localities (Shearer, 1993, 2001; Cai et al., 2006). About 500 species of meiosporic euascomycete species have been reported from freshwater and this group consists of species of Discomycetes, Loculoascomycetes and Pyrenomycetes in a proportion of 111:143:246 (Shearer et al., 2007).

Freshwater environments show wide variations in terms of their physical and chemical characteristics. The season of sampling is one of the main factors affecting colonization and diversity of freshwater fungi
(Cai et al., 2003; Luo et al., 2004; Nikolcheva and Bärlocher, 2005). Most of the fungal species can be collected throughout the year, but their richness and relative abundance are influenced by the seasonal availability of the woody substrates in temperate regions (Bärlocher, 1992 a, b, c). Seasonal changes in the temperature have been shown to effect fungal species richness and diversity (Suberkropp, 1984; Bärlocher et al., 2008; Luo et al., 2004). illustrated that differences in fungal communities and richness at different seasons were insignificant even though the summer and winter are distinct (high versus low rainfall) in the Kunming region (Southwest China).

Seasonal patterns and succession of fungi in freshwater habitat has been studied in many sectors of the world (Hughes, 1962; Alabi, 1971; Iqbal and Webster, 1973; Lamore and Goos, 1978; Suberkropp, 1984; Aimer and Segedin, 1985; Thomas et al., 1989, 1992; Khulbe, 1991; Shearer and Webster, 1991; Sridhar and Bärlocher, 1993; Fallah, 1999; Sivichai et al., 2000; Tsui et al., 2001; Ho et al., 2002; Luo et al., 2004).

In Egypt, while numerous investigations have been conducted dealing with the seasonal

* Corresponding author:

Dr. Ahmed M. Abdel-Raheem

$凶$ a.abdel-rahem@mailcity.com 
occurrence of zoosporic fungi and aquatic hyphomycetes in various water habitats (Abdel-Raheem, 1988, 1992; El-Hissy et al., 1982, 1990, 2000; Khalil et al., 1993, 1995) and myxomycetes (Saad-Elden, 2008). There are no studies were conducted on the seasonal fluctuation of higher freshwater fungi on decaying plant materials in Nile Delta region.

\section{Materials and Methods: \\ Study area:}

The studied area (Nile Delta) lies between latitude $30^{\circ} 08^{\prime} \mathrm{S}$ and $31^{\circ} 20^{\prime} \mathrm{N}$ and between longitudes $30^{\circ} 54^{\prime} \mathrm{W}$ and $31^{\circ} 45^{\prime} \mathrm{E}$ (Fig. 1). The Delta has a Mediterranean climate, characterized by little rainfall. The delta temperatures were averaging $30-48{ }^{\circ} \mathrm{C}$ and 10 $19{ }^{\circ} \mathrm{C}$ in summer and winter, respectively. Usually it is rains and humid in winter (Elewa, 2010).

Samples collection, preparation and examination:

Submerged and decaying plant substrates (3318 sample) were collected periodically from River Nile and irrigation canals in the period from February 2010 to December 2011. Cleaned samples were incubated at room temperature with the diffuse of light in moist chamber or clean transparent plastic bags. Cultures were sprayed by sterile distilled water from time to time to avoid dryness of the samples. Sterile water can be sprayed by using a fine aerosol spray. Samples examined periodically for about 4-6 months. Cultures were checked for sporulating structures using a dissecting microscope (Olympus SZ61 or model T12, CE Olympus Co, Ltd). Water Samples were collected for hydrochemical analysis in clean polyethylene bottles from most sampling sites in the four season's collections.

\section{Data analysis:}

Frequencies of occurrence of each species were calculated. Shannon-Weiner index (Shannon and Weaver, 1963) was applied to evaluate the diversities of freshwater ascomycetes in the different four seasons. The Jaccard's and Sørenson's similarity indices (Jaccard 1908; Sørenson, 1949). were used to compare the similarity of the species composition between the different seasons.
Calculations were carried out according to Magurran (1988).

1. Frequency of occurrence of each fungus (\%) was calculated on the following formula $=$ (Number of samples that a particular species occurred on / Total number of examined samples) x 100

2. Shannon-Weiner index ( $\alpha$-diversity) was calculated for each studied area by using Shannon's formula (Shannon and Weaver 1963).

$$
\left(H^{\prime}\right)=-\sum \mathrm{Pi} \log \mathrm{Pi},
$$

Where $\mathrm{Pi}$ is the relative abundance of a particular species (the proportion of the total number of individuals represented by species i).

3. Jaccard's and Sørenson's similarity indices were used to compare the similarity of the species composition between seasons collection. Sørenson's and Jaccard's similarity indices were calculated according to the formula:

Sørenson similarity index $(C s)=2 C /$ $(\mathrm{a}+\mathrm{b})$.

Jaccard similarity index $(\mathrm{Cj})=\mathrm{C} /$ $(a+b-c)$.

Where $\mathbf{C}=$ number of species common to both sites, $\mathbf{a}=$ total numbers of species in site $\mathbf{1}, \mathbf{b}$ total numbers of species in site 2. These indices are designed to equal 1 in absolute similarity and 0 if the sites are dissimilar and have no species in common.

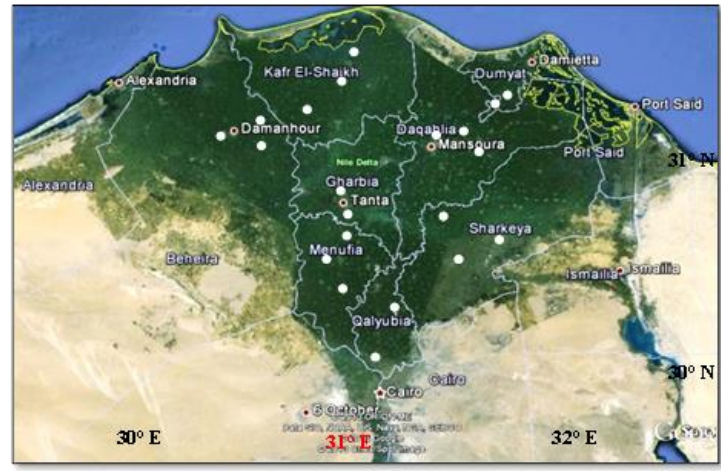

Fig. (1): A map showing the location of studied area in Nile Delta region

\section{Results:}

Seasonally changes in averages of physical and chemical parameters of water samples on the different season's collection are presented in table (1). One hundred and ten ascomycetes 
species representing 58 genera were identified from samples collected periodically from Nile Delta region in the period from February 2010 to December 2011. A list of species, frequency of occurrence of each species in the studied four seasons is given in table (2). Number of ascomycetes species was fluctuated between 53 (February 2010) and 67 (August 2010). Number of ascomycetes records was declined to the lowest value in February 2010 (311 records) and was high in May 2011 collection (552 records) as shown in table (3).

The most common ascomycetes species in Nile Delta region were Zopfiella latipes, reported on $(6.99 \%)$ of the total collected submerged samples and Microthelia sp. 1 (4.77), Helicascus aegyptiacus (2.49\%) and Naïs aquatica (2.46\%) (Table 2). Twenty-two (20\%) ascomycetes were collected throughout the entire year while, thirty-six species $(32.7 \%)$ were reported in one season without others. Number of unique species and list of exclusively reported ones in each season were listed in table (4).
Sordariomycetes and Dothideomycetes dominate the ascomycete assemblage in Delta region. The highest number of Sordariomycetes species (41 species) was reported in May 2011 collection while; the highest number of Dothideomycetes species (21 species) was in August collection (Fig. 2). Discomycetes was reported by only three species in May 2011. Zopfiella and Leptosphaeria were the most common genera in August 2010 collection, were represented by 159 and 44 records, respectively. Nais and Zopfiella were more common in May 2011 collection. Achaetomium and Kirschsteiniothelia were the most common genera in December 2011 collection (Table 2).

Some freshwater ascomycetes species were more common in particular seasons e.g. Zopfiella latipes was more common in August 2010 (16.5\% of the total collected samples), Naïs aquatica in May 2011 5.99, Microthlia sp. 1 in February 2010 (6.2\%), Helicascus aegyptiacus in December 2011 (5.36\%) (Table 2).

\begin{tabular}{|c|c|c|c|c|c|c|c|c|c|c|}
\hline \multirow[b]{2}{*}{ Seasons } & \multicolumn{3}{|c|}{ Physical properties } & \multicolumn{7}{|c|}{ Chemical propertiess } \\
\hline & $\begin{array}{l}\text { W.T } \\
{ }^{0} \mathrm{C}\end{array}$ & $\mathrm{pH}$ & $\begin{array}{l}\text { TDS } \\
\text { ppm }\end{array}$ & $\begin{array}{c}\mathrm{Cl}^{-} \\
\mathrm{ppm}\end{array}$ & $\begin{array}{l}\mathrm{Ca}^{2+} \\
\mathrm{Ppm}\end{array}$ & $\begin{array}{c}\mathrm{Mg}^{2+} \\
\mathrm{ppm}\end{array}$ & $\begin{array}{l}\mathrm{SO}_{4}{ }^{2-} \\
\mathrm{ppm}\end{array}$ & $\begin{array}{l}\mathrm{NO}_{2}^{-} \\
\mathrm{ppm}\end{array}$ & $\begin{array}{l}\mathrm{NH}_{3} \\
\mathrm{ppm}\end{array}$ & $\begin{array}{c}\mathrm{PO}_{4}{ }^{3-} \\
\mathrm{ppm}\end{array}$ \\
\hline February 2010 & $16-18$ & 6.9 & 308.3 & 17.84 & 39.8 & 20.4 & 33.7 & 0.02 & 0.31 & 0.13 \\
\hline August 2010 & $27-29$ & 6.3 & 353.8 & 44.9 & 42.6 & 22.5 & 27.1 & 0.06 & 1.17 & 0.17 \\
\hline May 2011 & $20-22$ & 7.46 & 184.3 & 31.21 & 32.5 & 19.5 & 25.2 & 0.03 & 0.21 & 0.07 \\
\hline December 2011 & $10-12$ & 7.5 & 345 & 42.5 & 32.8 & 17.3 & 39.0 & 0.05 & 0.2 & 0.04 \\
\hline
\end{tabular}

Table (1): averages of physico-chemical parameters on the different season's collection.

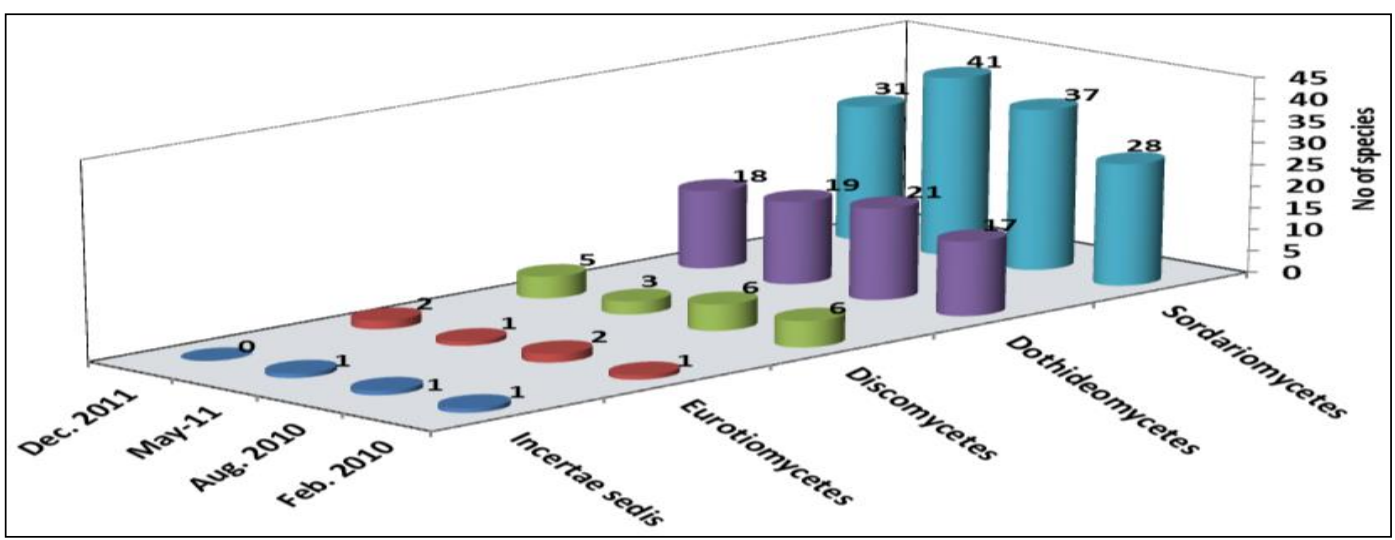

Fig. (2): Proportion of the ascomycetes classes in the studied seasons. 


\begin{tabular}{|c|c|c|c|c|c|}
\hline Species Name & Feb. 2010 & Aug. 2010 & May 2011 & Dec. 2011 & Total \\
\hline Achaetomium & 2.63 & 4.84 & 3.16 & 5.24 & 3.96 \\
\hline Achaetomium globosum JN Rai \& JP Tewari & 2.38 & 0.62 & - & 4.52 & 1.86 \\
\hline Achaetomium umbonatum K Rodr, Stchigel \& Guarro & - & - & - & 0.36 & 0.09 \\
\hline Achaetomium sp. & 0.25 & 4.22 & 3.16 & 0.36 & 2.01 \\
\hline $\begin{array}{l}\text { Aliquandostipite separans (Abdel-Wahab \& El-Shar.) J. Campb., Raja, A. } \\
\text { Ferrer, Sivichai \& Shearer }\end{array}$ & - & - & 0.45 & - & 0.12 \\
\hline Aniptodera & 2.51 & 1.74 & 4.52 & 1.19 & 2.52 \\
\hline $\begin{array}{l}\text { Aniptodera aquadulcis (SY Hsieh, HS. Chang \& EB Jones) J } \\
\text { Campb., JL Anderson \& Shearer }\end{array}$ & 0.88 & 0.50 & 2.15 & - & 0.9 \\
\hline Aniptodera chesapeakensis Shearer \& MA Mill. & 1.25 & 0.74 & 2.03 & 1.07 & 1.29 \\
\hline Aniptodera inflatiascigera KM Tsui, KD Hyde \& Hodgkiss Hodgkiss & - & 0.50 & 0.11 & - & 0.15 \\
\hline Aniptodera margarition Shearer & 0.13 & - & 0.23 & - & 0.09 \\
\hline Aniptodera sp. & 0.25 & - & - & 0.12 & 0.09 \\
\hline Annulatascus & - & 1.24 & 0.68 & 0.24 & 0.54 \\
\hline Annulatascus licualae J. Fröhl. \& KD Hyde & - & 0.62 & 0.11 & - & 0.18 \\
\hline Annulatascus nilensis Abdel-Wahab \& Abdel-Aziz & - & 0.12 & 0.23 & 0.24 & 0.15 \\
\hline Annulatascus palmietensis Goh, KD Hyde \& Steinke & - & 0.50 & - & - & 0.12 \\
\hline Annulatascus sp. & - & - & 0.34 & - & 0.09 \\
\hline Anthostomella sp. & 0.13 & - & - & - & 0.03 \\
\hline Apiosordaria terrestris (SC Jong and EE Davis) JC Krug, Udagawa \& & 0.13 & - & - & - & 0.03 \\
\hline Arnium mendax N Lundq. & - & - & 0.11 & - & 0.03 \\
\hline Ascobolus & 0.63 & 0.5 & 0.79 & 0.24 & 0.54 \\
\hline Ascobolus calesco AE Bell \& Mahoney & - & 0.25 & - & - & 0.06 \\
\hline Ascobolus castaneus Teng, Sinensia & 0.25 & - & - & 0.12 & 0.09 \\
\hline Ascobolus behnitziensis Kirschst. & 0.38 & 0.25 & 0.79 & 0.12 & 0.39 \\
\hline Astrosphaeriella aosimensis I. Hino \& Katum. & 0.25 & - & - & 0.24 & 0.12 \\
\hline Bombardia hyalina GS Verma & 0.38 & 0.12 & 1.24 & - & 0.45 \\
\hline Caryospora & 0.5 & 0.37 & 0.34 & 1.31 & 0.63 \\
\hline Caryospora sp. 1 & 0.25 & 0.37 & 0.34 & 1.19 & 0.54 \\
\hline Caryospora sp. 2 & 0.25 & - & - & 0.12 & 0.09 \\
\hline Cercophora costaricensis (GC Carroll \& Munk) O Hilber and R Hilber & - & 0.62 & - & - & 0.15 \\
\hline Chaetomium & 2.25 & 1.86 & 2.83 & 2.62 & 2.4 \\
\hline Chaetomium bostrychodes Zopf & - & 0.87 & 2.49 & - & 0.87 \\
\hline Chaetomium globosum Kunze & 1.5 & 0.37 & 0.11 & 0.95 & 0.72 \\
\hline Chaetomium sp. 1 & 0.75 & 0.25 & - & 1.67 & 0.66 \\
\hline Chaetomium sp. 2 & - & 0.37 & 0.23 & - & 0.15 \\
\hline Cosmospora sp. & - & 0.37 & 0.11 & - & 0.12 \\
\hline Emericella nidulans (Eidam) Vuill., & - & 0.37 & - & - & 0.09 \\
\hline Falciformispora sp. & - & - & 0.11 & - & 0.03 \\
\hline Halosarpheia heteroguttulata SW Wong, KD Hyde \& EB Jones & 1.5 & 1.36 & 0.57 & 0.36 & 0.93 \\
\hline Iodophanus & 0.63 & - & - & 0.36 & 0.24 \\
\hline Iodophanus carneus (Pers.) Korf & - & - & - & 0.36 & 0.09 \\
\hline Iodophanus testaceus (Moug.) Korf & 0.63 & - & - & - & 0.15 \\
\hline Jahnula & - & 0.49 & 0.9 & 0.36 & 0.45 \\
\hline
\end{tabular}

Table (2): Frequency of occurrence of ascomycetes species in the studied seasons. 


\begin{tabular}{|c|c|c|c|c|c|}
\hline Species Name & Feb. 2010 & Aug. 2010 & May 2011 & Dec. 2011 & Total \\
\hline Jahnula aquatica (Kirschst.) Kirschst. & - & - & 0.34 & 0.12 & 0.12 \\
\hline Jahnula australiensis K.D. Hyde & - & 0.12 & - & - & 0.03 \\
\hline Jahnula granulosa KD Hyde \& SW Wong & - & - & 0.11 & - & 0.03 \\
\hline Jahnula poonythii KD Hyde \& SW Wong & - & 0.12 & - & - & 0.03 \\
\hline Jahnula sangamonensis Shearer \& Raja & - & - & - & 0.12 & 0.03 \\
\hline Jahnula sp. $l$ & - & 0.25 & - & - & 0.06 \\
\hline Jahnula sp. 2 & - & - & 0.34 & 0.12 & 0.12 \\
\hline Jahnula sp. 3 & - & - & 0.11 & - & 0.03 \\
\hline Jobellisia viridifusca KM Tsui \& KD Hyde & - & - & - & 0.12 & 0.03 \\
\hline Helicascus aegyptiacus Abdel-Wahab \& Abdel-Aziz & 0.63 & 0.25 & 3.5 & 5.36 & 2.49 \\
\hline Leptosphaeria & 3.51 & 5.46 & 4.75 & 7.98 & 5.43 \\
\hline Leptosphaeria agnita & 3.38 & 0.62 & 1.70 & 2.98 & 2.16 \\
\hline Leptosphaeria xerophylli Ellis & - & 4.34 & - & - & 1.05 \\
\hline Leptosphaeria sp. & 0.13 & 0.5 & 3.05 & 5.0 & 2.22 \\
\hline Lindgomyces sp. & - & - & - & 0.24 & 0.06 \\
\hline Linocarpon $s p$ & - & - & 0.23 & 0.12 & 0.09 \\
\hline Lojkania dimidiata ZQ Yuan \& ME Barr & 0.25 & 0.37 & 0.23 & 0.36 & 0.3 \\
\hline Lophiostoma sp. & - & - & 2.03 & 2.14 & 1.08 \\
\hline Lophodermium sp. & 1.38 & 0.74 & 1.70 & 0.83 & 1.17 \\
\hline Lulworthia & 0.63 & - & 1.13 & 1.19 & 0.75 \\
\hline Lulworthia grandispora Meyers & 0.63 & - & 1.13 & 0.95 & 0.69 \\
\hline Lulworthia medusa var. biscaynia Meyers & - & - & - & 0.24 & 0.06 \\
\hline Massarina & 0.13 & - & 0.11 & - & 0.06 \\
\hline Massarina australiensis KD Hyde & 0.13 & - & - & - & 0.03 \\
\hline Massarina fluviatilis Aptroot \& Van Ryck. & - & - & 0.11 & - & 0.03 \\
\hline Microascus & 0.25 & 0.37 & 0.34 & 0.24 & 0.3 \\
\hline Microascus cinereus CA Fuentes \& FA Wolf & 0.25 & - & 0.23 & 0.12 & 0.15 \\
\hline Microascus trigonosporus CW Emmons \& BO Dodge & - & 0.37 & 0.11 & 0.12 & 0.15 \\
\hline $\begin{array}{l}\text { Micropeltopsis quinquecladiopsis EB Jones, Sivichai \& Hywel-Jones } \\
\text { Hywel-Jones }\end{array}$ & - & 0.37 & - & - & 0.09 \\
\hline Microthelia & 6.25 & 3.35 & 4.75 & 5.12 & 4.86 \\
\hline Microthelia sp. 1 & 6.25 & 3.35 & 4.75 & 4.76 & 4.77 \\
\hline Microthelia sp. 2 & - & - & - & 0.36 & 0.09 \\
\hline Minutisphaera fimbriatispora Shearer, AN Mill. \& A Ferrer & 0.38 & 0.50 & 0.34 & - & 0.3 \\
\hline Munkovalsaria donacina (Niessl) Aptroot & 0.13 & 0.50 & - & - & 0.15 \\
\hline Naïs aquatic KD Hyde & 0.25 & 1.61 & 5.99 & 1.67 & 2.46 \\
\hline Natantispora retorquens $\mathrm{C}$ (Shearer \& JL Crane) J.ampb, JL. & 3.0 & 0.87 & 3.96 & 1.43 & 2.34 \\
\hline Necteria $\mathrm{sp.}$ & - & - & 0.68 & - & 0.18 \\
\hline Neocosmospora haematococca (Berk. \& Broome) Nalim, & - & 0.25 & - & - & 0.06 \\
\hline Neomassariosphaeria sp. & - & - & - & 0.48 & 0.12 \\
\hline Neonectria sp. 1 & 0.25 & 0.25 & 0.57 & 0.48 & 0.39 \\
\hline Ophioceras & 1.88 & 0.37 & 1.47 & 1.2 & 1.23 \\
\hline Ophioceras commune Shearer, JL Crane \& W. Chen & 0.38 & 0.25 & 0.90 & 0.24 & 0.45 \\
\hline Ophioceras fusiforme Shearer, JL. Crane \& W. Chen & 1.25 & - & 0.57 & 0.36 & 0.54 \\
\hline $\begin{array}{l}\text { Ophioceras hongkongense KM Tsui, HY. Leung, KD Hyde } \\
\text { Hodgkiss Hodgkiss }\end{array}$ & 0.25 & 0.12 & - & 0.60 & 0.24 \\
\hline Orbilia & 1.25 & 0.75 & 0.11 & 0.12 & 0.54 \\
\hline Orbilia caudate Starbäck & - & 0.50 & 0.11 & - & 0.15 \\
\hline
\end{tabular}

Table (2) continued. 


\begin{tabular}{|c|c|c|c|c|c|}
\hline Species Name & Feb. 2010 & Aug. 2010 & May 2011 & Dec. 2011 & Total \\
\hline Orbilia sp. & 1.25 & 0.25 & - & 0.12 & 0.39 \\
\hline Phaeosphaeria & 1.26 & 1.12 & 1.02 & 0.6 & 0.99 \\
\hline Phaeosphaeria oryzae I. Miyake & 0.63 & - & 0.68 & - & 0.33 \\
\hline Phaeosphaeria typharum (Desm.) L. Holm & 0.25 & 0.50 & - & 0.60 & 0.33 \\
\hline Phaeosphaeria vagans (Niessl) OE Erikss. & 0.38 & 0.62 & 0.34 & - & 0.33 \\
\hline Pleoseptum sp. & 0.25 & - & - & - & 0.06 \\
\hline Pleospora & 0.63 & 0.75 & 1.13 & 2.26 & 1.2 \\
\hline Pleospora phaeocomoides (Berk. \& Broome) G. Winter & 0.25 & - & - & - & 0.06 \\
\hline Pleospora vagans $\mathrm{Niess}$ & - & 0.25 & 0.45 & 1.31 & 0.51 \\
\hline Pleospora sp. 1 & 0.38 & 0.25 & 0.68 & 0.95 & 0.57 \\
\hline Pleospora sp. 2 & - & 0.25 & - & - & 0.06 \\
\hline Podospora & 0.51 & 1.85 & 3.17 & 2.74 & 2.1 \\
\hline Podospora off. Pyriformis (B ay er) Cain & 0.25 & - & 0.57 & - & 0.21 \\
\hline Podospora communis (Speg.) Niess1 & - & 0.12 & 1.47 & - & 0.42 \\
\hline Podospora dolichopodalis JH Mirza \& Cain & 0.13 & 1.12 & 0.23 & 1.43 & 0.72 \\
\hline Podospora glutinans (Cain) Cain & - & 0.37 & - & - & 0.09 \\
\hline Podospora spinulosa RS Khan \& Cain & - & 0.12 & - & 1.31 & 0.36 \\
\hline Podospora sp. 1 & - & - & 0.9 & - & 0.24 \\
\hline Podospora sp. 2 & 0.13 & 0.12 & - & - & 0.06 \\
\hline Porosphaerellopsis sp. 1 & 0.13 & - & 0.34 & - & 0.12 \\
\hline Pseudohalonectria & 0.13 & 0.25 & 0.23 & 0.24 & 0.21 \\
\hline Pseudohalonectria lignicola Minoura \& T. Muroi & 0.13 & - & 0.23 & 0.24 & 0.15 \\
\hline Pseudohalonectria sp. 1 & - & 0.25 & - & - & 0.06 \\
\hline Rivulicola incrustata KD Hyde, in Hyde, Read, Jones \& Moss & 0.13 & - & - & 0.12 & 0.06 \\
\hline Roumegueriella rufula (Berk. \& Broome) Malloch \& Cain & - & - & - & 0.48 & 0.12 \\
\hline Saccobolus citrinus Boud. \& Torrend & 0.13 & 0.25 & - & - & 0.09 \\
\hline Savoryella & 0.13 & 0.24 & 0.22 & 0.48 & 0.27 \\
\hline Savoryella aquatica $\mathrm{KD} \mathrm{Hyde}$ & - & 0.12 & 0.11 & 0.24 & 0.12 \\
\hline Savoryella lignicola EB Jones \& RA Eaton & 0.13 & 0.12 & 0.11 & 0.24 & 0.15 \\
\hline Schizothecium sp. & - & - & 0.34 & 0.12 & 0.12 \\
\hline Sporormiella lata (Griffiths) S.I. Ahmed \& Cain & - & 0.12 & - & - & 0.03 \\
\hline Togninia minima (Tul. \& C. Tul.) Berl. & - & 0.25 & 0.11 & - & 0.09 \\
\hline Westerdykella & 0.25 & 1.74 & 1.02 & 2.74 & 1.44 \\
\hline Westerdykella dispersa (Clum) Cejp \& Milko & 0.25 & 1.49 & 1.02 & 2.74 & 1.38 \\
\hline Westerdykella multispora (Saito \& Minoura) Cejp \& Milko & - & 0.25 & - & - & 0.06 \\
\hline Zopfiella & 2.75 & 19.72 & 6.1 & 4.88 & 8.28 \\
\hline $\begin{array}{l}\text { Zopfiella cephalothecoidea Guarro, Abdullah, Al-Saadoon \& } \\
\text { Gené Gené }\end{array}$ & - & - & 0.34 & - & 0.09 \\
\hline Zopfiella karachiensis (S.I. Ahmed \& Asad) Guarro & - & 3.22 & 1.24 & 0.36 & 1.2 \\
\hline Zopfiella latipes (N. Lundq.) Malloch \& Cain & 2.75 & 16.5 & 4.52 & 4.52 & 6.99 \\
\hline Zygopleurage & - & $\mathbf{0 . 3 7}$ & 0.11 & - & 0.12 \\
\hline Zygopleurage multicaudata Mirza & - & 0.12 & 0.11 & - & 0.06 \\
\hline Zygopleurage zygospora (Speg.) Boedijn & - & 0.25 & - & - & 0.06 \\
\hline Unidentified Ascomycetes sp. 1 & - & 0.37 & 0.34 & - & 0.18 \\
\hline Unidentified Ascomycetes sp. 2 & 1.0 & 0.37 & 0.45 & 0.36 & 0.54 \\
\hline Unidentified Ascomycetes sp. 3 & - & 0.12 & 0.11 & - & 0.06 \\
\hline
\end{tabular}

Table (2) continued. 
$\mathrm{N}=$ Records number; $\mathrm{FO}=$ frequency of occurrence; FA= Frequency of appearance of each species in the studied seasons.

Temporal fungal distribution of freshwater fungi in Delta region patterns was difficult to be observed because many species were reported by single record and other many had a very low frequency of occurrence as shown in dominance-diversity curves (Fig. 3). Statistically, there was no significant differences between season's fungal communities $(\mathrm{P}=0.67)$ (using Kruskal-wallis one way analysis of variance) in Nile Delta region.

Shannon-Weiner indices were calculated based on the number of species and individuals. The results show that the differences in the fungal diversity among the four collections are insignificant. Species Shannon-Weiner indices ranged from 3.17 to 3.5 in the studied seasons. Summer collection had the lowest diversity value, while spring and autumn collection had the highest ones (Table 3).

\begin{tabular}{|l|c|c|c|c|}
\hline Diversity & Feb-10 & Aug-10 & May-11 & Dec-11 \\
\hline No of collected samples & 794 & 806 & 879 & 839 \\
\hline No. of ascomycetes species & 53 & 67 & 65 & 56 \\
\hline Ascomycetes Individuals & 311 & 463 & 552 & 472 \\
\hline Shannon-Wiener index & 3.3 & 3.17 & 3.5 & 3.35 \\
\hline
\end{tabular}

Table (3): Distribution of freshwater ascomycetes in studied season's collections.

Similarity indices were calculated among studied season's collections to evaluate the similarity of freshwater fungal communities at different times. Similarity matrix of Jaccard's and Sørensen's coefficient indexes revealed that there are no clear differences between fungal communities of seasonal collections (Table 5). Species similarity was greatest between December 2011 and February 2010 communities. Jaccard's and Sørensen's coefficient of similarity of these seasons was $47 \%$ and $64 \%$ respectively. The summer and autumn collections shared less similarity (Jaccard 34\%; Sørensen 51\%) as shown in table (5).

\begin{tabular}{|c|c|c|c|}
\hline February 2010 & August 2010 & May 2011 & December 2011 \\
\hline Anthostomella sp. & Annulatascus palmietensis & $\begin{array}{l}\text { Aliquandostipite } \\
\text { separans }\end{array}$ & $\begin{array}{l}\text { Achaetomium } \\
\text { umbonatum }\end{array}$ \\
\hline Apiosordaria terrestris & Ascobolus calesco & Annulatascus sp. & Jahnula sangamonensis \\
\hline Iodophanus testaceus & Cercophora costaricensis & Arnium mendax & Jobellisia viridifusca \\
\hline Massarina australiensis & Emericella nidulans & Falciformispora sp. & Lindgomyces sp. \\
\hline Pleoseptum sp. & Jahnula australiensis & Jahnula granulosa & Lulworthia medusa \\
\hline \multirow[t]{10}{*}{ Pleospora phaeocomoides } & Jahnula poonythii & Jahnula sp. 3 & Microthelia sp. 2 \\
\hline & Jahnula sp. l & Massarina fluviatilis & Neomassariosphaeria sp. \\
\hline & Leptosphaeria xerophylli & Necteria sp. & Roumegueriella rufula \\
\hline & Neocosmospora haematococca & Podospora sp. 1 & \\
\hline & Pleospora sp. 2 & Zopfiella cephalothecoidea & \\
\hline & Podospora glutinans & & \\
\hline & Pseudohalonectria sp. 1 & & \\
\hline & Sporormiella lata & & \\
\hline & Westerdykella multispora & & \\
\hline & Zygopleurage zygospora & & \\
\hline 6 & $\begin{array}{r}15 \\
\end{array}$ & 10 & 8 \\
\hline
\end{tabular}

Table (4): List of the unique ascomycetes species for each of the studied season's collections. 


\begin{tabular}{|c|c|c|c|c|}
\hline Season & Feb. 2010 & Aug. 2010 & May 2011 & Dec. 2011 \\
\hline Feb. 2010 & 1.00 & & & \\
\hline Aug. 2010 & $0.39(0.56)$ & 1.00 & & \\
\hline May. 2011 & $0.37(0.54)$ & $0.38(0.55)$ & 1.00 & \\
\hline Dec. 2011 & $0.47(0.64)$ & $0.34(0.51)$ & $0.39(0.56)$ & 1.00 \\
\hline
\end{tabular}

Table (5): Similarity matrix of Jaccard's and Sørensen's Coefficient indexes between seasons communities.

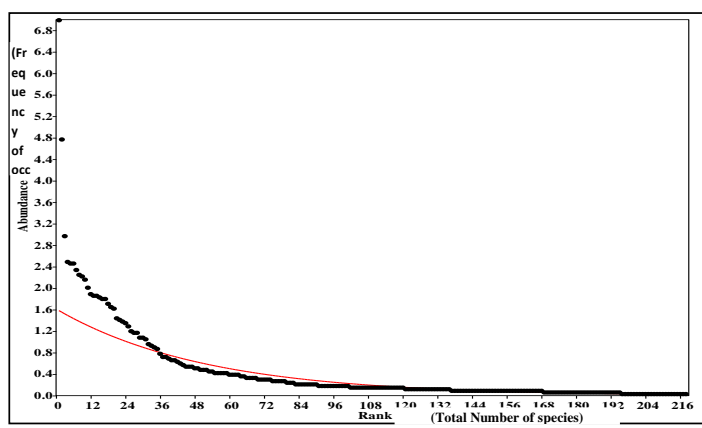

Fig. (3): Dominance diversity curves for total collected species in the different season in Nile Delta region

\section{Discussion}

A total of 110 ascomycetes species were identified from 3318 samples collected periodically from Delta region in the period from February 2010 and December 2011. In the current study we had high number and diversity of ascomycetes when compared with previous studies in Egypt or other countries. Differences in ascomycetes communities and species richness between the different seasonal collection in Nile delta region were statistically low significant. Twenty-two species of freshwater ascomycetes were collected throughout the entire year in Nile Delta region. The highest species richness, number of records was observed in August 2010 and May 2011 collections. Lamore and Goos (1978). noted that fungal species richness on naturally occurring wood samples submerged in a temperate stream was highest in September, following a period of heavy rainfall. Seasonal changes in the temperature have been shown to effect fungal species richness and diversity (Suberkropp 1984; Bärlocher et al., 2008). Fallah (1999) illustrated that species numbers of ascomycetes of north temperate lakes in Wisconsin were highest in May and June from 1995 to 1997 and the lowest numbers of fungi occurred in the months of January and February when the lakes were frozen.
Insignificant differences in diversity indexes between fungal communities of seasons were observed in this study and these similar to previous studies (Tsui et al., 2001; Sivichai et al., 2000). Ho et al., (2002) observed that higher species richness, fewer dominant fungi and more infrequent fungi were found on naturally occurring submerged wood during the hot wet season, as compared to the cool dry season in Tai Po Kau Forest Stream, Hong Kong.

Some common freshwater fungi species were dominant in specific seasons. Thirty six species of freshwater fungi in Nile Delta were reported by one record in winter collection. A large number of freshwater fungi recorded on submerged wood in this and previous studies are infrequent taxa (Shearer and Crane, 1986; Shearer and Webster, 1991).

The seasonal fluctuation of freshwater ascomycetes in Nile Delta region gets back to changes in climatic and water physicochemical parameters in the studied seasons. Water temperature reached to maximum of 29 ${ }^{0} \mathrm{C}$ on August 2010 collection, and was at 10 ${ }^{0} \mathrm{C}$ on December 2011. Concentration of ammonia, $\mathrm{NO}_{2}{ }^{-}$and $\mathrm{PO}_{4}{ }^{3-}$ was high in August 2010 collection. The aquatic fungal communities have been found to be effected by variations in temperature (Shearer, 1972; Iqbal and Webster, 1977; Suberkropp, 1984). Chamier (1992) reviewed a number of important factors that effect on the fungal communities inside the stream such as water temperature, conductivity, $\mathrm{pH}$, nitrate and phosphorus concentration. Furthermore, riparian vegetation had been regarded as an important factor influencing freshwater fungal communities (Fabre 1996; Tsui et al., 2000).

\section{References:}

Abdel-Raheem, A.M. (1988). Studies on aquatic hyphomycetes in the river Nile 
near Sohag. M. Sc. Thesis, Assiut University, $140 \mathrm{pp}$.

Abdel-Raheem, A.M. (1992). Studies on aquatic phycomycetes and hyphomycetes in Upper Egypt. $\mathrm{PhD}$ thesis, Assiut University, $320 \mathrm{p}$.

Aimer, R.D. and Segedin, B.P. (1985). Fluctuation in spore numbers of aquatic hyphomycetes in a New Zealand stream. Bot J Linn Soc. 91: 61-66.

Alabi, R.O. (1971). Factors affecting seasonal occurrence of Saprolegniaceae in Nigeria; Trans. Brit. Mycol. Soc. 56: 289-299.

Bärlocher, F. (1992a). Effects of Drying and Freezing Autumn Leaves on Leaching and Colonization by Aquatic Hyphomycetes. Freshwater Biol. 28 (1): 1-7.

Bärlocher, F. (1992b). Community organization. In: The ecology of aquatic hyphomycetes. Eds. F. Bärlocher. Springer-Verlag, Heidelberg, Berlin. pp. 38-76.

Bärlocher, F. (1992c). Research on aquatic hyphomycetes: Historical background and overview. In: The ecology of aquatic hyphomycetes. Ed. F. Bärlocher. Springer-verlag, Heidelberg, Berlin. pp. 1-15.

Bärlocher, F. Seena, S., Wilson, K.P. and Williams, D.D. (2008). Raised water temperature lowers diversity of hyporheic aquatic hyphomycetes. Freshwater Biol. 53 (2): 368-379.

Cai, L., Ji, K.F. and Hyde, K.D. (2006). Variation between freshwater and terrestrial fungal communities on decaying bamboo culms. Antonie van Leeuwenhoek. 89(2): 293-301.

Cai, L., Zhang, K.Q., McKenzie, E.H. and Hyde, K.D. (2003). Freshwater fungi from bamboo and wood submerged in the Liput River in the Philippines. Fungal Divers. 13: 1-12.

Chamier, A.C. (1992). Water chemistry. In: The ecology of aquatic hyphomycetes. Springer. 152-172.

Elewa, H.H. (2010). Potentialities of water resources pollution of the Nile River
Delta, Egypt. Open Hydrology Journal. 4: $1-13$

El-Hissy, F., El-Zayat, S. and Massoud, M. (2000). Monthly and vertical fluctuations of aquatic fungi at different depths in Aswan High Dam Lake, Egypt. Aquatic mycology across the millennium. 5: 165-173

El-Hissy, F., Moharram, A. and El-Zayat, S. (1990). Studies on the mycoflora of Aswan High Dam Lake, Egypt: Monthly variations. J Basic Microb. 30 (2): 81-94

El-Hissy, F.T., Moubasher, A.H. and EINagdy, M.A. (1982). Seasonal fluctuations of fresh water fungi in River Nile (Egypt). Zeitschrift fur Allgemeine Mikrobiologie. 22: 521-527.

Fabre, E. (1996). Relationships between aquatic hyphomycetes communities and riparian vegetation in 3 Pyrenean streams. C R Acad Sa Paris, Sa Vieme Science. 319: 107-111

Fallah, P.M. (1999). Ascomycetes from North temperate lakes in Wisconsin. $\mathrm{PhD}$ thesis, University of Illinois, UrbanaChampaign, USA, 190 pp.

Ho, W.H., Yanna, Hyde, K.D. and Hodgkiss, I.J. (2002). Seasonality and sequential occurrence of fungi on wood submerged in Tai Po Kau Forest Stream, Hong Kong. Fungal Diversity. 10: 21-43.

Hughes, G.C. (1962). Seasonal periodicity of the Saprolegniaceae in the south eastern United States; Trans. Br. MycoL Soc. 45: 519-531.

Iqbal, S.H. and Webster, J. (1973). Aquatic hyphomycetes spora of the River Exe and its tributaries. Trans Br Mycol Soc. 60: 331-346.

Iqbal, S.H. and Webster, J. (1977). Aquatic hyphomycete spora of some Dartmoor streams. Trans. br. mycol. Soc. 69: 233241.

Jaccard, P. (1908). Nouvelles resherches sur la distribution florale. Bull. Soc. Vaudoise Sci. Nat. 44: 233-270.

Jones, E.B.G. and Pang, K.L. (2012). Tropical aquatic fungi. Biodivers Conserv 21 (9): $2403-2423$. 
Khallil, A.M., El-Hissy, F.T. and AbdelRaheem, A.M. (1993). Monthly variations of Oomycetes (zoosporic fungi) and aquatic hyphomycetes at Sohag (Upper Egypt). Acta Societatis Botanicorum Poloniae. 62: 67-73.

Khallil, A.M., Elhissy, F.T. and Ali, E.H. (1995). Seasonal Fluctuations of Aquatic Fungi Recovered from Egyptian Soil (Delta Region). J Basic Microb. 35 (2): 93-102.

Khulbe, R.D. (1991). An ecological study of water molds of some rivers of Kumaun Himalayas. Indian Tropical Ecology. 32: 127-135.

Lamore, B.J. and Goos, R.D. (1978). Woodinhabiting fungi of a freshwater stream in Rhode Island. Mycologia. 1025-1034.

Luo, J., Yin, J.F., Cai, L., Zhang, K.Q. and Hyde, K.D. (2004). Freshwater fungi in Lake Dianchi, a heavily polluted lake in Yunnan, China. Fungal Divers. 16: 93112.

Magurran, A.E. (1988). Ecological diversity and its measurement. Princeton University Press, Englewood Cliffs, NJ.

McLaughlin, D.J., McLaughlin, E.G., Lemke, P.E. and eds. (2001). The Mycota VIIA and VIIB. Berlin: Springer-Verlag.

Nikolcheva, L.G. and Bärlocher, F. (2005). Seasonal and substrate preferences of fungi colonizing leaves in streams: traditional versus molecular evidence. Environ Microbiol. 7 (2): 270-280.

Saad-Eldin, M. (2008). Biodiversity and some physiological studies of Myxomycetes in Upper Egypt, MSc thesis. Sohag University, Egypt, 233 pp.

Shannon, C.E. and Weaver, W. (1963). The mathematical theory of communication. Univ. Illinois Press, Urbana, USA.

Shearer, C.A. (1972). Fungi of the Chesapeake Bay and its tributaries. III. The distribution of wood-inhabiting Ascomycetes and Fungi Imperfecti of the Patuxent River. Am J Bot. 961-969

Shearer, C.A. (1993). The Freshwater Ascomycetes. Nova Hedwigia. 56 (1-2): 1-33.

Shearer, C.A. (2001). The distribution of freshwater filamentous Ascomycetes. In
Trichomycetes and other Fungal Groups, Robert W. Lichtwardt Commemoration (eds. J.K. Misra and B.W. Horn). Science Publishers Inc. Enfield, New Hampshire. 225-292.

Shearer, C.A. and Crane, J. (1986). Illinois fungi. XII: Fungi and myxomycetes from wood and leaves submerged in southern Illinois swamps. Mycotaxon. 25 (2): 527-538.

Shearer, C.A. and Webster, J. (1991). Aquatic Hyphomycete Communities in the River Teign .4. Twig Colonization. Mycol Res. 95: 413-420.

Shearer, C.A., Descals, E., Kohlmeyer, B., Kohlmeyer, J., Marvanova, L., Padgett, D., Porter, D., Raja, H.A., Schmit, J.P., Thorton, H.A. and Voglymayr, H. (2007). Fungal biodiversity in aquatic habitats. Biodivers Conserv. 16 (1): 4967.

Sivichai, S., Jones, E. and Hywel-Jones, N. (2000). Fungal colonization of wood in a freshwater stream at Khao Yai National Park, Thailand. Fungal Divers. 5: 71-88

Sørensen, T. (1949). A method of establishing groups of equal amplitude in plant sociology based on similarity of species and its application to analyses of the vegetation of Danish commons. Biol. Skr. K. Danske Vidensk. Selsk. 5: 1-34.

Sridhar, K.R. and Bärlocher, F. (1993). Seasonal-Changes in Microbial Colonization of Fresh and Dried Leaves. Arch Hydrobiol. 128 (1): 1-12

Suberkropp, K. (1984). Effect of temperature on seasonal occurrence of aquatic Hyphomycetes. Transaction British Mycologia Society. 82: 53-62.

Thomas, K., Chilvers, G.A. and Norris, R.H. (1989). Seasonal Occurrence of Conidia of Aquatic Hyphomycetes (Fungi) in Lees Creek, Australian Capital Territory. Aust J Mar Fresh Res. 40 (1): 11-23.

Thomas, K., Chilvers, G.A. and Norris, R.H. (1992). Aquatic Hyphomycetes from Different Substrates-Substrate Preference and Seasonal Occurrence. Aust J Mar Fresh Res. 43 (2): 491-509. 
Tsui, C.K., Hyde, K.D. and Hodgkiss, I.J. (2000). Biodiversity of fungi on submerged wood in Hong Kong streams. Aquat Microb Ecol. 21 (3): 289-298.

Tsui, C.K., Hyde, K.D. and Hodgkiss, I.J. (2001). Longitudinal and temporal distribution of freshwater ascomycetes and dematiaceous hyphomycetes on submerged wood in the Lam Tsuen
River, Hong Kong. J N Am Benthol Soc. 20 (4):533-549.

Vijaykrishna, D., Jeewon, R. and Hyde, K.D. (2006). Molecular taxonomy, origins and evolution of freshwater ascomycetes. Fungal Diversity. 23 (1): 351-390.

\section{الملخص العربي}

\section{التنوع الموسمي للفطريات الزقيه في المياه العذبة بمنطقة دلتا النيل (مصر)}

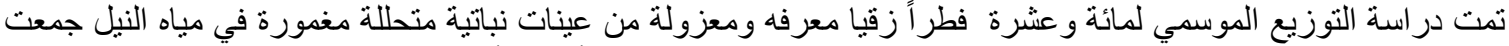

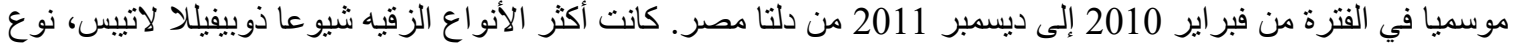

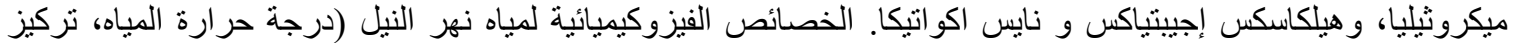

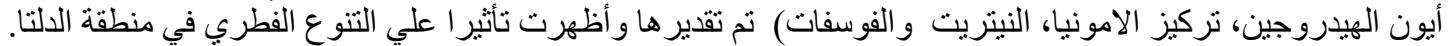

